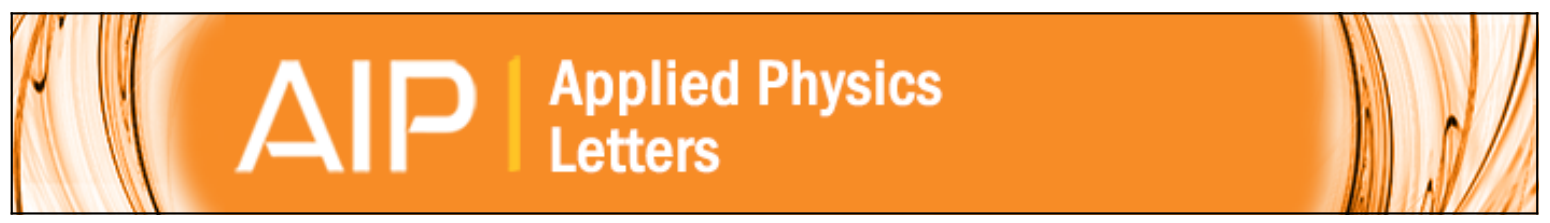

\title{
A gigahertz-range spin-wave filter composed of width-modulated nanostrip magnonic-crystal waveguides
}

Sang-Koog Kim, Ki-Suk Lee, and Dong-Soo Han

Citation: Applied Physics Letters 95, 082507 (2009); doi: 10.1063/1.3186782

View online: http://dx.doi.org/10.1063/1.3186782

View Table of Contents: http://scitation.aip.org/content/aip/journal/apl/95/8?ver=pdfcov

Published by the AIP Publishing

\section{Articles you may be interested in}

Position dependent spin wave spectrum in nanostrip magnonic waveguides

J. Appl. Phys. 115, 133906 (2014); 10.1063/1.4870448

Tuning the band structures of a one-dimensional width-modulated magnonic crystal by a transverse magnetic field

J. Appl. Phys. 115, 053904 (2014); 10.1063/1.4863776

Engineering spin-wave channels in submicrometer magnonic waveguides

AIP Advances 3, 032144 (2013); 10.1063/1.4799738

Spin wave localization and softening in rod-shaped magnonic crystals with different terminations

J. Appl. Phys. 112, 033911 (2012); 10.1063/1.4743003

Spin-wave propagation in a microstructured magnonic crystal

Appl. Phys. Lett. 95, 262508 (2009); 10.1063/1.3279138

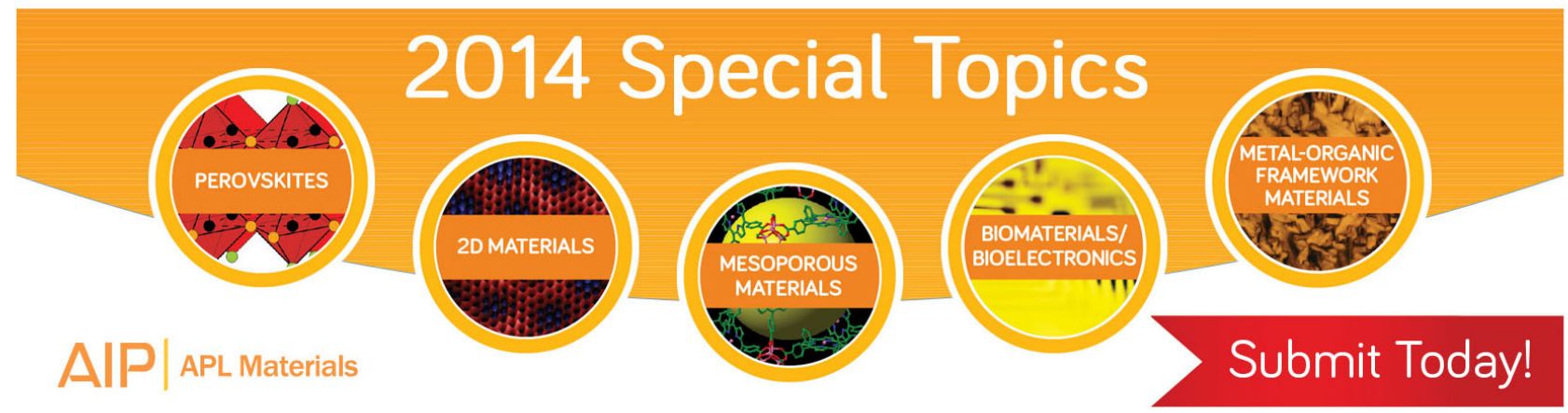




\title{
A gigahertz-range spin-wave filter composed of width-modulated nanostrip magnonic-crystal waveguides
}

\author{
Sang-Koog Kim, ${ }^{\text {a) }}$ Ki-Suk Lee, and Dong-Soo Han \\ Research Center for Spin Dynamics and Spin-Wave Devices, Nanospinics Laboratory, Research Institute \\ of Advanced Materials, Department of Materials Science and Engineering, Seoul National \\ University, Seoul 151-744, Republic of Korea
}

(Received 13 May 2009; accepted 1 July 2009; published online 26 August 2009)

\begin{abstract}
We found a robust magnonic-crystal waveguide structure for use as an efficient gigahertz-range spin-wave filter that passes only spin waves of chosen narrow band frequencies and filters out the other frequencies. The structure consists of the serial combinations of various width modulations with different periodicities and motifs in planar-patterned thin-film nanostrips composed of a single soft magnetic material. The observed magnonic band gaps result from both the translation symmetry of the one-dimensional width modulation and the higher-quantized width-mode spin waves excited from scattering at the periodic edge-steps of the width modulation. This work brings us one step closer to practical implementations of spin waves in information transmission and processing devices. (C) 2009 American Institute of Physics. [DOI: 10.1063/1.3186782]
\end{abstract}

Due to the current needs of ultrafast informationprocessing and ultrahigh-density information-storage devices, different types of nanoscale devices have been explored along with the recent developments in advanced nanofabrication technologies. A prominent example is spin waves (called magnons), which are collective excitations of individual spins in ordered magnets. Through the propagation of spin waves having many harmonic frequencies, information signals can be transmitted along patterned waveguides made of magnetic materials.

Up to now, many previous theoretical, numerical, and experimental studies have focused mostly on fundamentally understanding the eigenmodes of excited spin waves and their dispersion relations including their controllability in infinite model and micrometer-sized (or larger) real systems. ${ }^{1}$ These studies have also stimulated technological implementations of spin waves in information transmission and processing devices, such as logic gates, ${ }^{2,3}$ logic circuits, ${ }^{4}$ and filters. ${ }^{5,6}$ However, not only the reliable control of propagating spin waves along well-behaved waveguides, ${ }^{3,5-10}$ but waveguide miniaturization ${ }^{2,3,11,12}$ down to less than micrometer size, are further necessary for real applications of such spin-wave devices. ${ }^{12}$

In this letter, as an extension of our earlier study, ${ }^{5}$ we report on the discovery of a planar-patterned magnoniccrystal waveguide (MCWG) that can be used for a promising gigahertz-range broadband spin-wave filter. This filter is composed simply of various nanostrips of different width modulations serially connected and made only of a single soft magnetic material [e.g., permalloy (Py)]. The underlying physics of the occurrence of magnonic band gaps and their relation to the geometrical width modulations, additionally, were studied by micromagnetic numerical calculations.

In this study, we used $10 \mathrm{~nm}$ thick Py thin-film nanostrips for MCWGs. As seen in Fig. 1, the entire MCWG structure is composed of three parts. One is the widthmodulated strip in the middle and the other two are the 30

\footnotetext{
${ }^{\text {a) }}$ Author to whom correspondence should be addressed. Electronic mail: sangkoog@snu.ac.kr.
}

$\mathrm{nm}$ wide strips at both ends. The middle area, acting as a spin-wave filter, is made of a planar-patterned width modulation (here, e.g., $W_{1}=30$ and $W_{2}=24 \mathrm{~nm}$ ), with a periodicity $P=P_{1}+P_{2}$ and its repeating number $N$ (see the inset of Fig. 1). $P_{1}\left(P_{2}\right)$ corresponds to the segment length of the 30 (24) nm wide strip. This proposed MCWG is expected to yield scattering of initially propagating spin waves from the periodic edge-steps of the width modulations. The simulation code used here is the OOMMF of version 1.2a4 (Ref. 13), which employs the Landau-Lifshitz-Gilbert equation of motion. ${ }^{14}$ Additional descriptions of the present simulation are described in Ref. 15. To excite and inject the lowest-mode spin waves with frequencies, $f_{\mathrm{SW}}$, ranging from 0 to $100 \mathrm{GHz}$, we used a "sinc" function, ${ }^{5,16} H_{y}(t)$ $=H_{0}\left\{\sin \left[2 \pi \nu_{\mathbf{H}}\left(t-t_{0}\right)\right]\right\} / 2 \pi \nu_{\mathbf{H}}\left(t-t_{0}\right)$ with $H_{0}=1.0 \mathrm{~T}$ (Ref. 17) and $\nu_{\mathbf{H}}=100 \mathrm{GHz}$. This field was applied along the $y$ axis only to the left-end local area $\left(1.5 \times 30 \times 10 \mathrm{~nm}^{3}\right)$ indicated by the dark-gray color in Fig. 1 .

To explore how the planar-patterned structure affects magnonic band structures, frequency spectra and dispersion curves were plotted [Fig. 2(a)] for spin waves propagating along the $x$ axis, at the pass of $y=15 \mathrm{~nm}$, through the indicated different structures of MCWGs. They were obtained by conducting fast Fourier transforms (FFTs) of the temporal $M_{z} / M_{s}$ (the perpendicular component of the magnetization normalized by its magnitude) oscillations in the given nanostrip of $N=0,1,2,4$, or 12 . All of the dispersion curves are

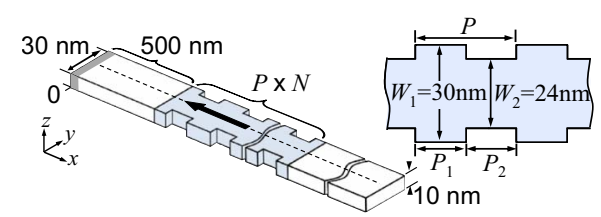

FIG. 1. (Color online) Schematic illustration of the model thin-film MCWG The equilibrium magnetizations point in the $-x$ direction in most areas, due to the shape anisotropy. The white-colored areas correspond to $30 \mathrm{~nm}$ wide nanostrips, and the blue-colored area represents the width-modulated part The left end indicated by the gray color is the local area for spin-wave excitation. The inset displays the two unit periods of the different width modulations. 
(a)
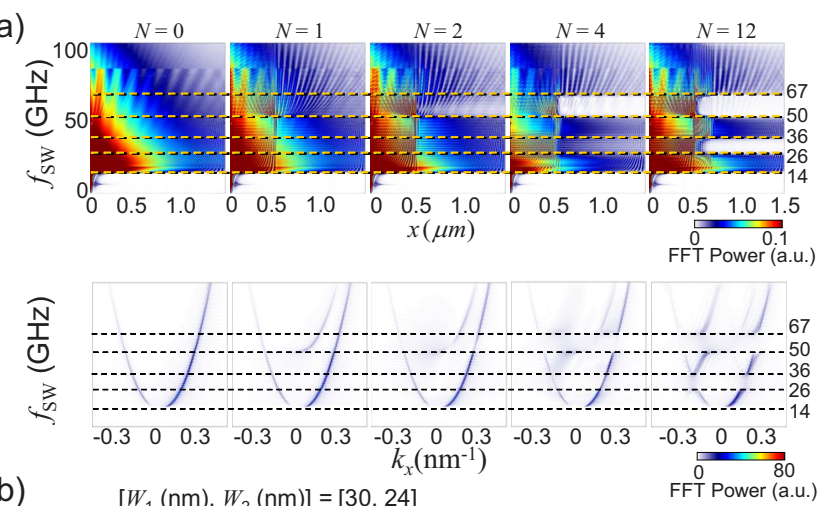

(b)

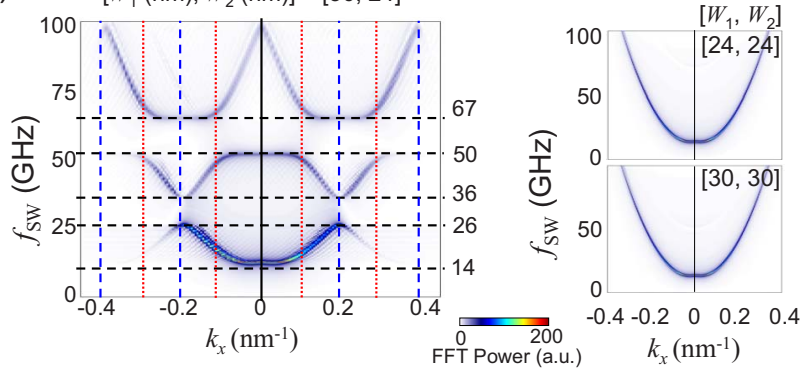

FIG. 2. (Color online) (a) Comparisons of frequency spectra (top) and dispersion curves (bottom) for a $30 \mathrm{~nm}$ wide nanostrip and the widthmodulated nanostrips of $\left[P_{1}(\mathrm{~nm}), P_{2}(\mathrm{~nm})\right]=[9,9]$ and $\left[W_{1}(\mathrm{~nm}), W_{2}(\mathrm{~nm})\right]$ $=[30,24]$ but with different $N$ values, as noted. (b) Dispersion curves of spin-wave excitation at the middle $(x=1000 \mathrm{~nm})$ of the indicated nanostrips of $N=55$.

nonsymmetric with respect to $k_{x}=0$, due to the excitation and injection of the lowest-mode spin waves from the left ends of the nanostrips. For $N=0$, there exists no forbidden band above $14 \mathrm{GHz}$, except for the intrinsic forbidden band below the potential barrier of $14 \mathrm{GHz}$, due to the nanostrip's width confinement. ${ }^{8,18,19}$ In contrast, for $N=2$ or 4 , two forbidden bands are observed. The higher band gap of $\Delta_{g}^{h}$ $=50-67 \mathrm{GHz}$ is clearer than the lower band gap of $\Delta_{g}^{\phi}$ $=26-36 \mathrm{GHz}$. As the value of $N$ increases further, both band gaps become more obvious. For example, the value of $N=12$ leads to a complex band structure and more distinct band gaps. To understand the role of the periodic width modulation in the occurrence of such complex band gaps, it is informative to investigate dispersion spectra obtained from the excitation and injection of spin waves from the middle of the indicated width-modulated MCWG and two single-width nanostrips (24 and $30 \mathrm{~nm}$ ) [Fig. 2(b)]. All of the dispersion spectra show the intrinsic forbidden bands below $14 \mathrm{GHz}$, due to the strip-width confinements. ${ }^{8,18,19}$ As seen in the left panel of Fig. 2(b), the lower band gaps occur at the positions $k_{x}=n \pi / P($ where $n=0, \pm 1, \pm 2, \ldots$, and $P=18 \mathrm{~nm})$, i.e., the Brillion zone (BZ) boundaries represented by the blue dashed vertical lines. This indicates that the lower band gaps are associated with the one-dimensional (1D) translation symmetry of the width modulation along the longitudinal direction, which yields interference between the initially propagating lowest-mode spin waves and their reflected waves from the edge-steps. In contrast, the higher band gaps are observed at $k_{x}=(2 n+1) \pi / P \pm \Lambda \mathrm{nm}^{-1}, \Lambda=0.08$ (denoted by the red dotted vertical lines), which are away from the BZ boundaries. The variations in $W_{1} / W_{2}$ as well as $P$ affect the numerical value of $\Lambda$ (Ref. 20). This reveals that the higher bands cannot be understood only through the 1D approach. As seen in the dispersion curve of $N=1$ [Fig. 2(a)], the ad-

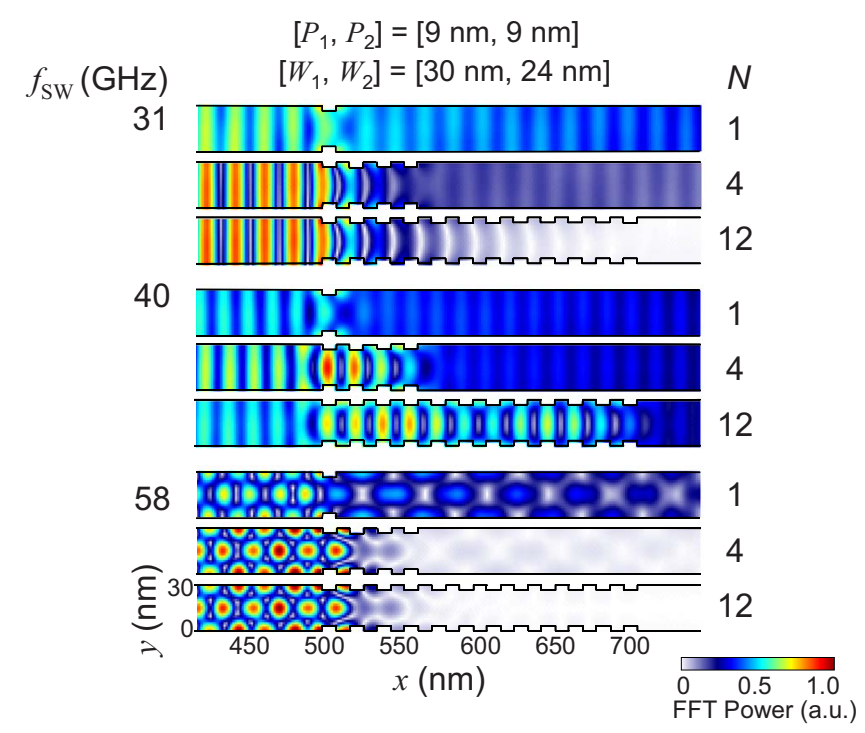

FIG. 3. (Color online) Plane-view color-coded images of the standing spinwave patterns obtained from the FFTs of the spatial distributions of the temporal $M_{z} / M_{s}$ evolution in the width-modulated nanostrips with the indicated different $N$ values for the specific frequencies noted.

ditional branch above $50 \mathrm{GHz}$ is caused by the 3rd-quantized width mode excited through the scattering of the lowest mode at the edge-steps. ${ }^{5,8,18,19}$ Consequently, the higher band gap results from the interaction between the higher-quantized width mode and the initial lowest-mode spin waves. ${ }^{5}$ The different physical origins of the occurrences of the higher and lower band gaps are also understood by the distinct standing wave patterns shown in Fig. 3 for three specific values of $f_{\mathrm{SW}}=31,58$, and $40 \mathrm{GHz}$, which are selected from among the forbidden and allowed bands. For $f_{\mathrm{SW}}=31 \mathrm{GHz}$, the nodes of standing waves in the regions before the widthmodulated strips are positioned parallel to the width direction. For $f_{\mathrm{SW}}=58 \mathrm{GHz}$, by contrast, the nodes are positioned in the transverse direction as well as the longitudinal direction.

According to the above findings, one can readily expect that magnonic band structures vary by $P, N, P_{1} / P_{2}$, and $W_{1} / W_{2}$. Figure 4(a) shows some examples of frequency spectra obtained from MCWGs with the indicated different periodic modulations. For the case of $P_{1}=P_{2}=7.5 \mathrm{~nm}$, the two wide band gaps of $\Delta_{g}=31-48$ and $\Delta_{g}=52-75 \mathrm{GHz}$ appear, and for $P_{1}=P_{2}=10.5 \mathrm{~nm}$, the relatively narrow band gaps of $\Delta_{g}=22-30$ and $\Delta_{g}=48-62 \mathrm{GHz}$ are found. For $P_{1}$ $=18$ and $P_{2}=3 \mathrm{~nm}$, in contrast, four narrow band gaps are observed. To construct more valuable maps on the variations in magnonic band gaps by both $P$ and $P_{1} / P$ (here the ratio of $W_{1} / W_{2}$ is kept constant; see Ref. 20 ), we conducted additional simulations with different nanostrips of various width modulations, i.e., variations in $P$ while retaining $P_{1}=P_{2}$ and of $P_{1} / P$ while maintaining $P=30 \mathrm{~nm}$. Figure 4(b) shows the results, where all of the gray regions correspond to the allowed bands. The white regions correspond to the forbidden bands obtained at $k_{x}=n \pi / P$, and the blue regions correspond to those observed away from the BZ boundaries. As seen in Fig. 4(b), the number of band gaps and the center and width of the individual gaps change dramatically with $P$. For the forbidden bands denoted by the white-color-coded region, the center position and width of each band decrease with the incressing $P$, whereas the center positions do not change 
(a) $\left[P_{1}(\mathrm{~nm}), P_{2}(\mathrm{~nm})\right] \times N$ $[7.5,7.5] \times 67$

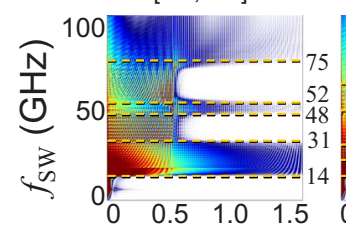

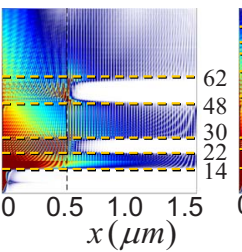

$[10.5,10.5] \times 48$
$[18,3] \times 48$

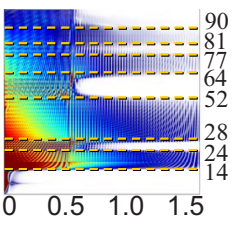

(b)

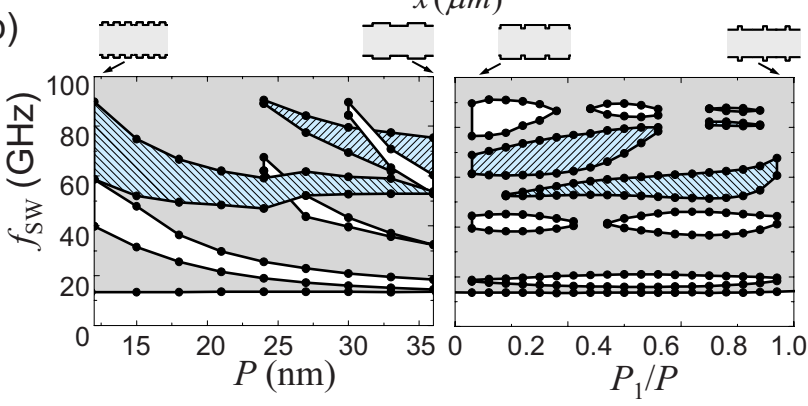

FIG. 4. (Color online) (a) Frequency spectra for the indicted nanostrips of $\left[W_{1}(\mathrm{~nm}), W_{2}(\mathrm{~nm})\right]=[30,24]$. (b) Band gap diagram with respect to $P$ while maintaining $P_{1}=P_{2}$ (left) and $P_{1} / P$ while maintaining $P=P_{1}+P_{2}=30 \mathrm{~nm}$ (right). The gray-color region indicates the allowed bands. The white- and blue-color regions denote the forbidden bands.

with $P_{1} / P$. For the forbidden bands marked by the bluecolor-coded region, by contrast, the positions slightly move with both $P$ and $P_{1} / P$. Consequently, Fig. 4(b) shows the fact that the number, position, and gap width of the forbidden bands can be manipulated readily by changing both $P$ and $P_{1} / P$.

Moreover, one can design a different type of efficient spin-wave filter that passes only the frequencies of spin waves in a chosen narrow band, filtering out most of the others. This proposed structure is of a simple planar thin film consisting of the serial combinations of various widthmodulated nanostrips of different periodicities, motifs, and

(a)

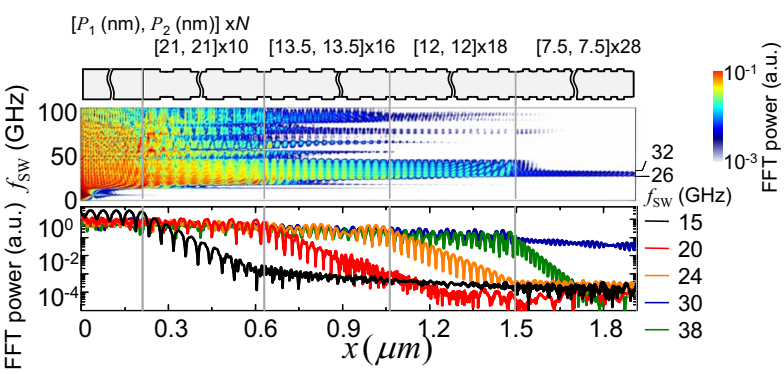

(b)

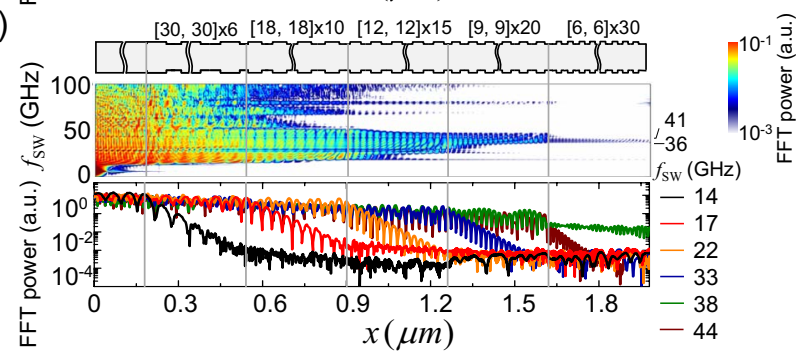

FIG. 5. (Color online) In the first, second, and third rows of (a) and (b), two different planar structures of serially connected width-modulated nanostrips, the frequency spectra of propagating spin waves along the $x$ axis at $y$ $=15 \mathrm{~nm}$ in the corresponding MCWGs, and the FFT power profiles vs $x$ for the indicated frequencies, are illustrated, respectively. repeating numbers. Figures 5(a) and 5(b) show two examples of such planar MCWGs that were designed to pass only spin waves of $\Delta f_{\mathrm{SW}}=26-32 \mathrm{GHz}$ and $\Delta f_{\mathrm{SW}}=36-41 \mathrm{GHz}$, respectively, according to the band gap maps shown in Fig. 4(b). The frequency spectra versus the propagation distance shown in the second rows exhibit the fact that the spin waves having the two designed pass bands of 26-32 and 36-41 GHz propagate well through the corresponding MCWG. Furthermore, the FFT power profiles for the specific $f_{\text {Sw }}$ values evidence that the spin wave amplitudes are reduced only by $10 \%$ even after traveling the distance as far as $1.0 \mu \mathrm{m}$ (see the third rows). Such frequency-controllable, narrow-passband spin-wave filters operating in the gigahertz range provide a promising future for information-processing devices.

This work was supported by Basic Science Research Program through the National Research Foundation of Korea (NRF) funded by the Ministry of Education, Science, and Technology (Grant No. 20090063589).

${ }^{1}$ Spin Dynamics in Confined Magnetic Structures III, edited by B. Hillebrands and A. Thiavilles (Springer, Berlin, 2006).

${ }^{2}$ M. P. Kostylev, A. A. Serga, T. Schneider, B. Leven, and B. Hillebrands, Appl. Phys. Lett. 87, 153501 (2005); T. Schneider, A. A. Serga, B. Leven, B. Hillebrands, R. L. Stamps, and M. P. Kostylev, ibid. 92, 022505 (2008).

${ }^{3}$ K.-S. Lee and S.-K. Kim, J. Appl. Phys. 104, 053909 (2008).

${ }^{4}$ A. Khitun, D. E. Nikonov, M. Bao, K. Galatsis, and K. L. Wang, Nanotechnology 18, 465202 (2007).

${ }^{5}$ K.-S. Lee, D.-S. Han, and S.-K. Kim, Phys. Rev. Lett. 102, 127202 (2009).

${ }^{6}$ A. V. Chumak, A. A. Serga, B. Hillebrands, and M. P. Kostylev, Appl. Phys. Lett. 93, 022508 (2008).

${ }^{7}$ R. Hertel, W. Wulfhekel, and J. Kirschner, Phys. Rev. Lett. 93, 257202 (2004).

${ }^{8}$ S. Choi, K.-S. Lee, K. Y. Guslienko, and S.-K. Kim, Phys. Rev. Lett. 98 , 087205 (2007).

${ }^{9}$ V. V. Kruglyak and R. J. Hickena, J. Magn. Magn. Mater. 306, 191 (2006).

${ }^{10}$ S. Choi, K.-S. Lee, and S.-K. Kim, Appl. Phys. Lett. 89, 062501 (2006); S.-K. Kim, S. Choi, K.-S. Lee, D.-S. Han, D.-E. Jung, and Y.-S. Choi, ibid. 92, 212501 (2008).

${ }^{11}$ A. A. Serga, T. Neumann, A. V. Chumak, and B. Hillebrands, Appl. Phys. Lett. 94, 112501 (2009).

${ }^{12}$ S. V. Vasiliev, V. V. Kruglyak, M. L. Sokolovskii, and A. N. Kuchko, J. Appl. Phys. 101, 113919 (2007).

${ }^{13} \mathrm{~A}$ version of the OOMMF code used is $1.2 \mathrm{a} 4$. See http://math.nist.gov/ oommf.

${ }^{14}$ L. D. Landau and E. M. Lifshitz, Phys. Z. Sowjetunion 8, 153 (1935); T. L. Gilbert, Phys. Rev. 100, 1243 (1955).

${ }^{15}$ The chosen material parameters corresponding to Py are as follows: the saturation magnetization $M_{s}=8.6 \times 10^{5} \mathrm{~A} / \mathrm{m}$, the exchange stiffness $A_{\mathrm{ex}}$ $=1.3 \times 10^{-11} \mathrm{~J} / \mathrm{m}$, the damping constant $\alpha=0.01$, and the gyromagnetic ratio $\gamma=2.21 \times 10^{5} \mathrm{~m} /$ As with zero magnetocrystalline anisotropy. The size of the unit cell is $1.5 \times 1.5 \times 10 \mathrm{~nm}^{3}$.

${ }^{16}$ D.-S. Han, S.-K. Kim, J.-Y. Lee, S. J. Hermsdoerfer, H. Schultheiss, B. Leven, and B. Hillebrands, Appl. Phys. Lett. 94, 112502 (2009).

${ }^{17}$ Such high field strength was used to obtain large amplitudes of emitted spin waves. However much smaller fields, e.g., 10 and 100 Oe, also result in the same band structures.

${ }^{18}$ T. W. O'Keeffe and R. W. Patterson, J. Appl. Phys. 49, 4886 (1978); V. E. Demidov and S. O. Demokritov, Phys. Rev. B 77, 064406 (2008); K. Yu. Guslienko, S. O. Demokritov, B. Hilbrands, and A. N. Slavin, ibid. 66 , 132402 (2002).

${ }^{19}$ M. P. Kostylev, G. Gubbiotti, J.-G. Hu, G. Carlotti, T. Ono, and R. L. Stamps, Phys. Rev. B 76, 054422 (2007).

${ }^{20}$ The $W_{1} / W_{2}$ ratio can affect the excitation efficiency of the highly quantized width modes and hence result in changes in the width and position of the higher band gaps. 